\title{
A Privacy-Preserving Buyer-Seller Watermarking Protocol Based on Priced Oblivious Transfer
}

\author{
Alfredo Rial, Josep Balasch, and Bart Preneel, Member, IEEE
}

\begin{abstract}
Buyer-seller watermarking protocols allow copyright protection of digital goods. To protect privacy, some of those protocols provide buyers with anonymity. However, anonymous e-commerce protocols pose several disadvantages, like hindering customer management or requiring anonymous payment mechanisms. Additionally, no existing buyer-seller watermarking protocol provides fair exchange.

We propose a novel approach for the design of privacypreserving buyer-seller watermarking protocols. In our approach, the seller authenticates buyers but does not learn which items are purchased. Since buyers are not anonymous, customer management is eased and currently deployed methods of payment can be utilized.

We define an ideal functionality for privacy-preserving copyright protection protocols. To realize our functionality, a protocol must ensure that buyers pay the right price without disclosing the purchased item, and that sellers are able to identify buyers that released pirated copies. We construct a protocol based on priced oblivious transfer and on existing techniques for asymmetric watermark embedding. Furthermore, we implement and evaluate the efficiency of our protocol, and we explain how to extend it in order to achieve optimistic fair exchange.
\end{abstract}

Index Terms-Buyer-Seller Watermarking Protocol, Priced Oblivious Transfer, Fair Exchange.

\section{INTRODUCTION}

The rapid development of communication networks has led to a situation that facilitates online e-commerce of digital goods. However, it also poses threats to copyright protection and to customers' privacy. On the one hand, distribution of illegal copies is eased, and thus mechanisms that allow the protection of intellectual property rights are needed. On the other hand, information about which items are bought can reveal sensitive data about the buyer. This information can easily be shared among service providers to create personal profiles. Consequently, privacy concerns discourage online ecommerce [1], and regulations to enforce privacy protection are being promulgated [2].

Previous work. Fingerprinting schemes deter people from illegally redistributing digital copies by enabling the seller

Copyright (c) 2010 IEEE. Personal use of this material is permitted. However, permission to use this material for any other purposes must be obtained from the IEEE by sending a request to pubs-permissions@ieee.org.

This work was supported in part by the Research Council K.U.Leuven: GOA TENSE, and by the IAP Programme P6/26 BCRYPT of the Belgian State (Belgian Science Policy). The work of A. Rial was supported by the Research Foundation - Flanders (FWO). Josep Balasch is funded by a $\mathrm{PhD}$ grant within the covenant between K.U.Leuven and R.U.Nijmegen universities.

Alfredo Rial, Josep Balasch, and Bart Preneel are with IBBT and the COSIC group of Departement Elektrotechniek (ESAT), Katholieke Universiteit Leuven, Kasteelpark Arenberg 10, B-3001, Leuven, Belgium (phone: +32 (0)16 32 96 06, fax:+32 (0)16 3219 69, e-mail: firstname.lastname@esat.kuleuven.be). of the data to identify the buyer. A scheme is said to be collusion-resistant [3] when it prevents a collusion of buyers up to a maximum size from producing non-traceable copies. In asymmetric fingerprinting schemes [4], the fingerprinted copy is only known to the buyer at the end of the purchase protocol. Thanks to this property, when the seller finds a redistributed copy, he can present it as a proof of buyer's misbehavior, and the buyer cannot claim that the copy was produced by the seller. In order to protect privacy, fingerprinting protocols that provide buyers with anonymity have been proposed [5].

Buyer-seller watermarking protocols [6] are asymmetric fingerprinting schemes in which the fingerprint is embedded by means of watermarking techniques. The basic idea is that each buyer obtains a slightly different copy of the digital content. Such difference, the watermark, does not harm the quality of the copy and cannot be removed by the buyer. Some buyer-seller watermarking protocols also provide buyers with anonymity [7], [8], [9].

As noted in [10], anonymous e-commerce protocols have several disadvantages. First, they hinder customer management. For example, the seller cannot give discounts to regular buyers or apply other loyalty marketing techniques. Second, they have to be used together with anonymous payment protocols (e.g. anonymous e-cash [11]), which makes it impossible the use of currently deployed payment protocols. Finally, they require the use of an underlying anonymous communication network, such as Tor [12]. It is well-known that achieving strong anonymity in such networks is a difficult goal [13]. Furthermore, some applications allow side-channel attacks against anonymity. For example, in location-based services, the service provider learns customer's location, and this information can be used to identify the a priori anonymous customer [14].

Additionally, e-commerce protocols are usually analyzed in order to prove their fairness [15]. Roughly speaking, fair exchange ensures that, at the end of the transaction, either the seller receives the payment and the buyer receives the purchased item, or both parties receive nothing. However, to the best of our knowledge, no fair buyer-seller watermarking protocol has been proposed.

Our contribution. We propose a different approach to provide privacy protection in buyer-seller watermarking protocols. In our approach, based on oblivious e-commerce protocols, buyers are authenticated by the seller, but the seller does not learn which items are purchased. This overcomes the disadvantages of anonymous purchase. Since buyers are authenticated, customer management is eased and currently deployed methods of payment can be utilized. As possible disadvantages, one can argue that the seller can find it difficult 
to learn which items are more demanded, e.g., to adapt his catalogue of products or to pay copyright owners. However, as noted in [10], this information can be obtained by other means, e.g., by conducting marketing researches.

We define formally privacy-preserving buyer-seller watermarking protocols, i.e., buyer-seller watermarking protocols in which the seller does not learn which items are purchased. We also provide a construction of such a protocol based on existing techniques for asymmetric watermark embedding and on priced oblivious transfer. (Priced oblivious transfer is the key building block of oblivious e-commerce protocols.) Finally, we explain how to extend our protocol to provide fair exchange.

Outline of the paper. In Section II we recall the definition of priced oblivious transfer and we define privacy-preserving buyer-seller watermarking protocols. We recall the definition of watermarking and of other cryptographic building blocks utilized in our construction in Section III. In Section IV we describe our construction, and we analyze its security in Section V. Additionally, we explain how to extend our construction to achieve fairness in Section VI, and we discuss its efficiency in Section VII. Finally, Section VIII draws a conclusion and discusses future work.

\section{DEFINITIONS}

\section{A. Security Model}

We define security following the ideal-world/real-world paradigm [16]. In the real world, a set of parties interact according to the protocol description in the presence of a real adversary $\mathcal{A}$, while in the ideal world dummy parties interact with an ideal functionality that carries out the desired task in the presence of an ideal adversary $\mathcal{E}$. A protocol $\psi$ is secure if there exists no environment $\mathcal{Z}$ that can distinguish whether it is interacting with adversary $\mathcal{A}$ and parties running protocol $\psi$ or with the ideal process for carrying out the desired task, where ideal adversary $\mathcal{E}$ and dummy parties interact with an ideal functionality $\mathcal{F}_{\psi}$. More formally, we say that protocol $\psi$ emulates the ideal process when, for any adversary $\mathcal{A}$, there exists a simulator $\mathcal{E}$ such that for all environments $\mathcal{Z}$, the ensembles $\operatorname{IDEAL}_{\mathcal{F}_{\psi}, \mathcal{E}, \mathcal{Z}}$ and $\operatorname{REAL}_{\psi, \mathcal{A}, \mathcal{Z}}$ are computationally indistinguishable. By applying the universal composition theorem [16], a protocol $\psi$ that realizes functionality $\mathcal{F}_{\psi}$ remains secure even when it is composed along with an unbounded number of protocol instances controlled by the adversary. We refer to [16] for a description of how these ensembles are constructed. Every functionality and every protocol invocation should be instantiated with a unique sessionID that distinguishes it from other instantiations. For the sake of ease of notation, we omit session-IDs from the description of our ideal functionalities.

\section{B. Priced Oblivious Transfer}

POT [17] is a two-party protocol between a seller $\mathcal{S}$ and a buyer $\mathcal{B}$, where $\mathcal{S}$ sells a set of messages $m_{1}, \ldots, m_{N}$ with prices $p_{1}, \ldots, p_{N}$ to $\mathcal{B}$. At each purchase, $\mathcal{B}$ chooses $\tau \in$ $\{1, \ldots, N\}$, gets $m_{\tau}$ and pays $p_{\tau} . \mathcal{S}$ must learn neither $\tau$ nor $p_{\tau}$, while $\mathcal{B}$ must not learn anything about the other messages.
We recall the ideal functionality $\mathcal{F}_{\mathrm{POT}}$ for priced oblivious transfer in [10].

\section{Functionality $\mathcal{F}_{\mathrm{POT}}$}

Parameterized with the number of messages $N$, the message length $l$, the maximum price $p_{\max }$, and the maximum deposit $D_{\max }$, and running with a seller $\mathcal{S}$ and buyers $\mathcal{B}_{1}, \ldots, \mathcal{B}_{n}, \mathcal{F}_{\text {POT }}$ works as follows:

- On input a message (init, $\left.m_{1}, p_{1}, \ldots, m_{N}, p_{N}\right)$ from $\mathcal{S}$, where each $m_{i} \in\{0,1\}^{l}$ and each $p \in$ $\left[0, p_{\max }\right]$, it stores $\left(m_{1}, p_{1}, \ldots, m_{N}, p_{N}\right)$ and sends (init, $\left.p_{1}, \ldots, p_{N}\right)$ to each buyer $\mathcal{B}$.

- On input a message (deposit, $a c_{i}$ ) from $\mathcal{B}_{i}$, where $a c_{i} \in\left[0, D_{\max }\right]$, if a (init, ..) message was not received before, then it does nothing. Otherwise, it stores $\left(\mathcal{B}_{i}, a c_{i}\right)$ and sends $\left(\right.$ deposit, $\left.\mathcal{B}_{i}, a c_{i}\right)$ to $\mathcal{S}$.

- On input a message (request, $\tau$ ) from $\mathcal{B}_{i}$, where $\tau \in[1, N]$, if message (init, ..) was not received, (deposit, $a c_{i}$ ) was not sent by $\mathcal{B}_{i}$ before or $a c_{i}-$ $p_{\tau}<0$, then it does nothing. Otherwise, it sends (request, $\mathcal{B}_{i}$ ) to $\mathcal{S}$. If $\mathcal{S}$ is corrupted, $\mathcal{F}_{\text {POT }}$ receives a bit (response, $b$ ) from $\mathcal{E}$, and otherwise $\mathcal{F}_{\text {POT }}$ sets $b=1$. If $b=0$, it sends (response, $\perp$ ) to $\mathcal{B}_{i}$. If $b=1$, it updates $a c_{i}=a c_{i}-p_{\tau}$ and sends (response, $m_{\tau}$ ) to $\mathcal{B}_{i}$.

\section{Privacy-Preserving Copyright Protection Protocol}

We describe an ideal functionality $\mathcal{F}_{\mathrm{PCP}}$ that models the behavior and desirable properties of any privacy-preserving copyright protection protocol, i.e., a copyright protection protocol in which buyers do not disclose to the seller which items are bought. We consider a setting with three parties: a seller $\mathcal{S}$ that sells protected messages $y$; a set of buyers $\mathcal{B}_{1}, \ldots, \mathcal{B}_{n}$ that purchase protected messages from $\mathcal{S}$; and a judge $\mathcal{J}$ that decides whether a buyer is guilty of releasing pirated copies. $\mathcal{F}_{\text {PCP }}$ is parameterized with a set of parties $\mathcal{P}$ that contains the aforementioned entities.

$\mathcal{F}_{\text {PCP }}$ models the properties that the protocol should fulfill under three assumptions. First, the judge $\mathcal{J}$ is never corrupted by the ideal adversary $\mathcal{E}$. Second, parties can be corrupted statically, i.e., the ideal adversary $\mathcal{E}$ decides at the beginning of the protocol execution the set of parties it wishes to corrupt and cannot modify this set throughout the execution. Finally, $\mathcal{F}_{\mathrm{PCP}}$ assumes that uncorrupted buyers never release pirated copies.

Under those assumptions, $\mathcal{F}_{\mathrm{PCP}}$ requires that buyers, after making an initial deposit, purchase items from the seller by disclosing neither the item bought nor the amount of money paid. This requirement must hold both when the seller is honest and when he is corrupted.

In addition, when the seller is uncorrupted, buyers receive a unique protected message $y$ at each purchase. This unique protected message, when released as a pirated copy, can be traced back to the corrupted buyer that released it. In our 
construction, unique protected messages are computed by embedding different watermarks into the original content.

When the seller $\mathcal{S}$ is corrupted, $\mathcal{F}_{\mathrm{PCP}}$ does not require the seller to send unique protected messages $y$. However, it requires that $\mathcal{S}$ is not able to frame uncorrupted buyers, who by assumption do not release pirated copies.

Below we describe formally $\mathcal{F}_{\mathrm{PCP}}$. In Section $\mathrm{V}$ we prove that our privacy-preserving buyer-seller watermarking protocol realizes functionality $\mathcal{F}_{\mathrm{PCP}}$. This means that our protocol fulfills the aforementioned properties.

\section{Functionality $\mathcal{F}_{\mathrm{PCP}}$}

Parameterized with the number of messages $N$, the message length $l$, the maximum price $p_{\max }$, and the maximum deposit $D_{\max }$, and running with a seller $\mathcal{S}$, a judge $\mathcal{J}$ and buyers $\mathcal{B}_{1}, \ldots, \mathcal{B}_{n}, \mathcal{F}_{\mathrm{PCP}}$ works as follows:

- On input a message (init, $m_{1}, p_{1}, \ldots, m_{N}, p_{N}$ ) from $\mathcal{S}$, it checks that each $m_{i} \in\{0,1\}^{l}$ and each $p \in$ $\left[0, p_{\max }\right]$. For each buyer $\mathcal{B}_{i}$, it computes a unique protected message $y_{i j}$ from message $m_{j}(j \in[1, N])$. If $\mathcal{S}$ is corrupted, $\mathcal{F}_{\mathrm{PCP}}$ receives (init, $\left\{\mathcal{B}_{i}, y_{i 1}\right.$, $\left.\left.\ldots, y_{i N}\right\}_{i=1}^{n}, p_{1}, \ldots, p_{N}\right)$ from the ideal adversary $\mathcal{E}$. $\mathcal{F}_{\mathrm{PCP}}$ stores $\left(\left\{\mathcal{B}_{i}, y_{i 1}, \ldots, y_{i N}\right\}_{i=1}^{n}, p_{1}, \ldots, p_{N}\right)$ and sends (init, $p_{1}, \ldots, p_{N}$ ) to each buyer $\mathcal{B}$.

- On input a message (deposit, $a c_{i}$ ) from $\mathcal{B}_{i}$, where $a c_{i} \in\left[0, D_{\max }\right]$, if a (init,...) message was not received before, then it does nothing. Otherwise, it stores $\left(\mathcal{B}_{i}, a c_{i}, d\right)$, where $d=1$, and sends (deposit, $\left.\mathcal{B}_{i}, a c_{i}\right)$ to $\mathcal{S}$.

- On input a message (request, $\tau$ ) from $\mathcal{B}_{i}$, where $\tau \in[1, N]$, if message (init,...) was not received, (deposit, $a c_{i}$ ) was not sent by $\mathcal{B}_{i}$ before or $a c_{i}-$ $p_{\tau}<0$, then it does nothing. Otherwise, it sends (request, $\mathcal{B}_{i}$ ) to $\mathcal{S}$. If $\mathcal{S}$ is corrupted, $\mathcal{F}_{\text {POT }}$ receives a bit (response, $b$ ) from $\mathcal{E}$, and otherwise $\mathcal{F}_{\text {POT }}$ sets $b=1$. If $b=0$, it sends (response, $\perp$ ) to $\mathcal{B}_{i}$. If $b=1$, it updates $a c_{i}=a c_{i}-p_{\tau}$ and sends (response, $y_{i \tau}$ ) to $\mathcal{B}_{i}$.

- On input a message (release, $y$ ) from the ideal adversary $\mathcal{E}, \mathcal{F}_{\mathrm{PCP}}$ searches for $y$ in the tuples $\left\{\mathcal{B}_{i}, y_{i 1}\right.$, $\left.\ldots, y_{i N}\right\}_{i=1}^{n}$ and, if found in the tuple of $\mathcal{B}_{i}$, sets $d$ to 0 in $\left(\mathcal{B}_{i}, a c_{i}, d\right)$. Otherwise $\mathcal{F}_{\mathrm{PCP}}$ stores $(\mathcal{E}, y)$.

- On input a message (detect,y), if $y$ belongs to a buyer $\mathcal{B}_{i}$ such that $d=1$ in the tuple $\left(\mathcal{B}_{i}, a c_{i}, d\right)$, $\mathcal{F}_{\mathrm{PCP}}$ sends (detresp, $\mathcal{B}_{i}$, not guilty) to $\mathcal{J}$ and $\mathcal{S}$, and otherwise it sends (detresp, $\mathcal{B}_{i}$, guilty) to $\mathcal{J}$ and $\mathcal{S}$. If $y$ is stored in a tuple $(\mathcal{E}, \ldots)$, it sends (detresp, $\mathcal{E}$, guilty) to $\mathcal{J}$ and $\mathcal{S}$.

Informally speaking, the requirements a protocol should fulfill in order to realize functionality $\mathcal{F}_{\mathrm{PCP}}$ can be summarized as follows:

- Correctness. The protocol should terminate successfully whenever its parties are honest.

- Traceability. Upon finding a pirated copy, $\mathcal{S}$ should always be able to trace and identify the buyer $\mathcal{B}$ that released it.

- Non-frameability. An honest buyer cannot be found guilty of releasing pirated copies.

- Direct Non-repudiation. A guilty buyer cannot deny she released the pirated copy. Additionally, $\mathcal{S}$ can convince a third party that $\mathcal{B}$ is guilty without needing interaction with $\mathcal{B}$.

- Privacy. $\mathcal{S}$ does not learn which contents are purchased.

- Anti-fraud. $\mathcal{S}$ is assured that $\mathcal{B}$ pays the right price and that $\mathcal{B}$ cannot purchase when running out of funds.

\section{Registration Authority}

In Section $\mathrm{V}$ we prove that our buyer-seller watermarking protocol realizes functionality $\mathcal{F}_{\mathrm{PCP}}$ in the $\mathcal{F}_{\mathrm{REG}}$-hybrid model, where parties register their public keys at a trusted registration entity. Below we depict the ideal functionality $\mathcal{F}_{\text {REG }}$ given in [16]. $\mathcal{F}_{\text {REG }}$ is parameterized with a set of participants $\mathcal{P}$, which is restricted to contain the buyers $\mathcal{B}_{1}, \ldots, \mathcal{B}_{n}$, the seller $\mathcal{S}$ and the judge $\mathcal{J}$. $\mathcal{F}_{\text {REG }}$ can be implemented with a public key infrastructure.

\section{Functionality $\mathcal{F}_{\text {REG }}$}

Parameterized with a set of parties $\mathcal{P}, \mathcal{F}_{\text {REG }}$ works as follows:

- Upon receiving (register, $v$ ) from party $P \in \mathcal{P}$, it records the value $(P, v)$.

- Upon receiving (retrieve, $P$ ) from party $P^{\prime} \in \mathcal{P}$, if $(P, v)$ is recorded then return (retrieve, $P, v)$ to $P^{\prime}$. Otherwise send (retrieve, $P, \perp$ ) to $P^{\prime}$.

\section{TEChNiCAl Preliminaries}

We recall the definition of watermarking and of other cryptographic building blocks utilized in our construction. Some subsections and notation utilized here and in the following sections is taken verbatim from [18].

\section{A. Blind Watermarking}

A blind and readable watermarking scheme [19] consists of a setup algorithm WATsetup, a watermark embedding algorithm WATemb and a watermark detection algorithm WATdet. WATsetup outputs a secret watermarking key $s w k$, a message space $\mathcal{M}$ and a watermark space $\mathcal{W}$. WATemb $(s w k, m, w)$, on input $s w k$, message $m \in \mathcal{M}$, and watermark $w \in \mathcal{W}$, outputs a watermarked message $y$. The algorithm WATemb can be computed in the encrypted domain, where both $w$ and the result $y$ are encrypted with a public key of a public key encryption scheme. The algorithm WATdet $(s w k, y)$ outputs the watermark $w$ embedded in $y$.

A secure watermarking scheme should be robust and collusion resistant. Let $d$ be a distortion metric that quantifies the distortion suffered by a watermarked content $y$ when it underwent signal processing operations such as compression, filtering, noise addition, desynchronization, cropping, insertions, 
mosaicing, and collage. Let $y^{\prime}$ be a distorted content. The robustness property requires that under a distortion metric $d$ and a distortion bound $D$, given $s w k$ output by WATsetup and $y$ output by WATemb $(s w k, m, w)$, WATdet $\left(s w k, y^{\prime}\right)$ outputs $w$ with overwhelming probability if $d\left(y, y^{\prime}\right) \leq D$.

The collusion resistance property [20] requires that a collusion of up to $l$ parties cannot manipulate or remove the watermark from a watermarked content by comparing or composing their differently watermarked copies. This property can be formalized as follows:

Definition 1 (Collusion Resistant Watermarking). The collusion resistance property is defined through the following game between a challenger $\mathcal{C}$ and an adversary $\mathcal{A}$.

- Challenge. $\mathcal{C}$ runs WATsetup to get swk, picks random original content $m \in \mathcal{M}$, and, for $i=1$ to l, picks random watermark $w_{i} \in \mathcal{W}$ and runs $y_{i}=\operatorname{WATemb}(s w k$, $\left.m, w_{i}\right)$. $\mathcal{C}$ sends $\left(y_{1}, \ldots, y_{l}\right)$ to $\mathcal{A}$.

- Response. $\mathcal{A}$ outputs watermarked content $y^{\prime}$.

$\mathcal{A}$ wins if there exists $i \in[1, l]$ such that $d\left(y_{i}, y^{\prime}\right) \leq D$ and WATdet $\left(s w k, y^{\prime}\right)$ outputs a watermark $w^{\prime}$ such that, for $i=1$ to $l, w^{\prime} \neq w_{i}$. A blind watermarking scheme is $l$ collusion resistant if all p.p.t. adversaries $\mathcal{A}$ win the game above with negligible probability.

Current practical watermarking schemes do not provide collusion-resistance against any p.p.t. adversary. In Section V we assume that the watermarking scheme used to instantiate the protocol fulfills this definition, and thus we conclude that our protocol is secure against any p.p.t. adversary. When the protocol is instantiated with a concrete watermarking scheme, the security offered against malicious buyers is lowered to the security offered by the watermarking scheme.

\section{B. Signature Schemes}

A signature scheme consists of the algorithms Keygen, Sign and VerifySig. Keygen outputs a secret key $s k$ and a public key $p k$. Sign $(s k, m)$ outputs a signature $s$ of message $m$. VerifySig $(p k, m, s)$ outputs accept if $s$ is a valid signature of $m$ and reject otherwise. A signature scheme must be correct and unforgeable [21]. Informally speaking, correctness implies that the VerifySig algorithm always accepts an honestly generated signature. Existential unforgeability means that no p.p.t. adversary should be able to output a message-signature pair $(s, m)$ unless he has previously obtained a signature on $m$. We can employ any existentially unforgeable signature scheme to instantiate the signature scheme (Keygen, Sign, VerifySig) employed by buyers in our construction in Section IV.

\section{Homomorphic Encryption}

A public key encryption scheme consists of the algorithms Keygen, Enc and Dec. Keygen outputs a public key $p k$ and a secret key $s k$. Enc outputs a ciphertext $c$ on input a public key $p k$ and a message $m$. Dec outputs the message $m$ on input the ciphertext $c$ and the secret key $s k$. Roughly speaking, indistinguishability under chosen plaintext attack [22] (INDCPA) guarantees that an adversary does not get any knowledge about $m$ from $c$.
We employ a homomorphic public key encryption scheme that supports two operations. An operation $\odot$ that, on input two ciphertexts $\operatorname{Enc}(p k, x)$ and $\operatorname{Enc}(p k, y)$ that encrypt messages $x$ and $y$, outputs a ciphertext $\operatorname{Enc}(p k, x+y)=\operatorname{Enc}(p k, x) \odot$ $\operatorname{Enc}(p k, y)$ that encrypts the addition of the messages, and an operation $\otimes$ that, on input a message $x$ and a ciphertext $\operatorname{Enc}(p k, y)$, outputs a ciphertext $\operatorname{Enc}(p k, x y)=x \otimes \operatorname{Enc}(p k, y)$ that encrypts the multiplication of the messages $x$ and $y$. The homomorphic public key encryption scheme proposed by Paillier [23], and its generalization by Damgård and Jurik [24], support these operations, and therefore can be used to instantiate the encryption scheme (BKeygen, BEnc, BDec) employed in Section IV.

In our construction in Section IV, we need a function that, on input a bit $b$ and an encryption $\operatorname{Enc}\left(p k, b^{\prime}\right)$ of a bit $b^{\prime}$, computes the encryption $\operatorname{Enc}\left(p k, b \oplus b^{\prime}\right)$, where $\oplus$ denotes the exclusive or operation. This function can be computed as follows. If $b=0$, output $\operatorname{Enc}\left(p k, b^{\prime}\right)$. If $b=1$, output $\operatorname{Enc}(p k, b) \odot\left(-1 \otimes \operatorname{Enc}\left(p k, b^{\prime}\right)\right)$.

\section{Zero-Knowledge Proofs of Knowledge}

A zero-knowledge proof of knowledge [25] is a two-party protocol between a prover and a verifier. The prover proves to the verifier knowledge of some secret input that fulfills some statement without disclosing this input to the verifier. The protocol should fulfill two properties. First, it should be a proof of knowledge, i.e., a prover without the knowledge of the secret input convinces the verifier with negligible probability. More technically, there exists a knowledge extractor that extracts the secret input from a successful prover with all but negligible probability. Second, it should be zero-knowledge, i.e., the verifier does not learn any information about the secret input. More technically, for all possible verifiers there exists a simulator that, without knowledge of the secret input, yields a transcript that cannot be distinguished from the interaction with a real prover.

To express a zero-knowledge proof of knowledge, we follow the notation introduced by Camenisch and Stadler [26]. For example, $\operatorname{PK}\{(x): y=f(x)\}$ denotes a "zero-knowledge proof of knowledge of secret input $x$ such that $y=f(x)$ ". Letters in the parenthesis, in this example $x$, denote the secret input, while $y$ and the function $f$ are also known to the verifier.

We employ a proof of knowledge PK $\left\{\left(s k^{\prime}\right):\left(p k^{\prime}, s k^{\prime}\right) \leftarrow\right.$ $\left.\operatorname{BKeygen}\left(1^{k}\right) \wedge C \leftarrow \operatorname{Enc}\left(p k, s k^{\prime}\right)\right\}$, i.e., a proof that $C$ is a correct encryption under $p k$ of the secret key $s k^{\prime}$ related with public key $p k^{\prime}$, so that a party in possession of the secret key $s k$ related with $p k$ can recover $s k^{\prime}$ from $C$. The verifiable encryption schemes proposed by Camenisch et al. [27] and by Poupard and Stern [28], which are provided with such a proof of knowledge, can be employed to instantiate the encryption scheme (JKeygen, JEnc, JDec) used in our construction in Section IV.

We also use a proof of knowledge of the statement $\operatorname{PK}\{(b)$ : $c \leftarrow \operatorname{Enc}(p k, b) \wedge b \in\{0,1\}\}$, i.e., a proof that the value $b$ encrypted in ciphertext $c$ under public key $p k$ is a bit. Such a proof is described in [24]. 


\section{Privacy-Preserving Buyer-SEller WATERMARKING PROTOCOL}

\section{A. Intuition Behind Our Construction}

Our privacy-preserving buyer-seller watermarking (PBSW) protocol is based on priced oblivious transfer (POT). POT allows buyers to purchase messages from the seller without the seller learning which messages are bought (see Section II). Existing secure POT schemes follow an assisted decryption approach in which the interaction between a seller $\mathcal{S}$ and a buyer $\mathcal{B}$ is divided into an initialization phase and several purchase phases. In the initialization phase, $\mathcal{S}$ encrypts the messages to be sold and sends the ciphertexts to $\mathcal{B}$. In each purchase phase, $\mathcal{S}$ helps $\mathcal{B}$ to decrypt one of the ciphertexts via an interactive protocol.

To allow for payments, existing POT schemes employ a prepaid mechanism. In the initialization phase, $\mathcal{B}$ makes an initial deposit to $\mathcal{S}$, and, in each purchase phase, $\mathcal{B}$ debits the price of the message from the deposit and proves to $\mathcal{S}$ that the remaining deposit is non-negative. $\mathcal{S}$ is able to verify those facts by learning neither the price of the message nor the new value of the deposit. We note that, to achieve full privacy, the initial deposit should be higher than the price of the most expensive item. Additionally, it is possible for the buyer to hide when she is buying something by having the seller include a dummy item with price zero.

Our PBSW protocol combines POT with existing techniques for asymmetric watermark embedding. In particular, we use a simplified version of the buyer-seller watermarking protocol in [18], in which buyers are not provided with anonymity. This protocol employs homomorphic encryption to allow $\mathcal{S}$ and $\mathcal{B}$ to jointly compute an encryption (with the public key of $\mathcal{B}$ ) of the watermark to be embedded in the message, in such a way that none of the parties knows the watermark. This prevents both a malicious seller from releasing pirated copies with the same watermark in order to frame the buyer, and a malicious buyer that releases pirated copies from invoking the possibility of being framed by the seller.

Additionally, the protocol involves a judge $\mathcal{J}$ to resolve disputes between $\mathcal{S}$ and $\mathcal{B}$. To this end, $\mathcal{B}$ sends to $\mathcal{S}$ a key escrow ciphertext that encrypts her secret key with the public key of the judge $\mathcal{J}$. When $\mathcal{S}$ accuses $\mathcal{B}$ of releasing pirated copies, $\mathcal{S}$ sends this ciphertext to $\mathcal{J}$ so that $\mathcal{J}$ can recover $\mathcal{B}$ 's secret key. With the secret key, $\mathcal{J}$ can check whether $\mathcal{B}$ is guilty or not guilty.

Our PBSW protocol consists of four phases: setup, initialization, purchase and arbitration. In the setup phase, $\mathcal{B}$ and $\mathcal{J}$ create key pairs and register their public keys with the registration authority. $\mathcal{S}$ computes a secret watermarking key.

In the initialization phase, $\mathcal{S}$ and $\mathcal{B}$ run an interactive protocol for asymmetric watermark embedding. As result, $\mathcal{S}$ obtains a set of $N$ watermarks, encrypted with the public key of $\mathcal{B}$, to be embedded in each of the $N$ messages. Each watermark is of the form $w=\phi \|\left(W_{\mathcal{S}} \oplus W_{\mathcal{B}}\right)$, where $W_{\mathcal{S}}$ and $W_{\mathcal{B}}$ are randomly chosen by $\mathcal{S}$ and $\mathcal{B}$ respectively, and $\phi$ is a random string, unique for each released content, chosen by $\mathcal{S}$. By using signal processing in the encrypted domain techniques, $\mathcal{S}$ embeds the watermarks and obtains $N$ encrypted watermarked messages. These watermarked messages are given as input to the seller initialization algorithm of the POT scheme, which produces $N$ ciphertexts to be sent to $\mathcal{B}{ }^{1}$ After that, the initialization phase follows that of the POT scheme.

The purchase phases follow those of the POT scheme, except that as result $\mathcal{B}$ obtains a watermarked message encrypted with her public key. $\mathcal{B}$ decrypts the result to obtain the watermarked message. In the arbitration phase, when $\mathcal{S}$ claims to have found a pirated copy, $\mathcal{S}$ employs the watermark detection algorithm to obtain the watermark $\phi \|\left(W_{\mathcal{S}} \oplus W_{\mathcal{B}}\right)$. $\mathcal{S}$ sends $\mathcal{J}$ the identity of the alleged pirate, i.e., the buyer $\mathcal{B}$ that received the content whose watermark included $\phi . \mathcal{J}$ checks whether $\mathcal{B}$ is guilty or not guilty.

Our construction employs authenticated channels. In its description we employ a single watermarking key swk. If, in order for the watermarking scheme to be collusion resistant, a different watermarking key should be associated to each of the original contents, our construction can be modified to watermark each content with a different key.

\section{B. A Syntax for POT Schemes}

Our construction can be instantiated with any POT scheme that realizes $\mathcal{F}_{\text {POT }}$, such as [10], [29]. Following the syntax for POT proposed in [29], a POT scheme consists of the following algorithms.

- A seller initialization algorithm POTInitS $\left(1^{\kappa}, m_{1}, p_{1}\right.$, $\left.\ldots, m_{N}, p_{N}, D_{\max }\right)$ that, on input the security parameter $1^{\kappa}$, message-price pairs $\left(m_{1}, p_{1}, \ldots, m_{N}, p_{N}\right)$ and the maximum deposit $D_{\max }$, outputs a secret key $s k_{\mathcal{S}}$, a public key $p k_{\mathcal{S}}$ and a set of $N$ ciphertexts $T$.

- A buyer initialization algorithm POTInitB $\left(1^{\kappa}, p k_{\mathcal{S}}, T\right.$, $\left.a c_{0}\right)$ that, on input the public key $p k_{\mathcal{S}}$, the ciphertexts $T$ and the buyer's deposit $a c_{0}$, outputs a payment message $P$ and buyer's state information $D_{0}^{\prime}$.

- An algorithm POTGetDep $\left(s k_{\mathcal{S}}, P, D_{\max }\right)$ that, on input the secret key $s k_{\mathcal{S}}$, the payment message $P$ and the maximum deposit $D_{\max }$, outputs the buyer's deposit $a c_{0}$ and seller's state information $D_{0}$.

- An algorithm $\operatorname{POTReq}\left(p k_{\mathcal{S}}, T, D_{i-1}^{\prime}, \tau\right)$ that, on input the public key $p k_{\mathcal{S}}$, the ciphertexts $T$, buyer's state information $D_{i-1}^{\prime}$ and selection value $\tau$, computes the request $Q$ for the $i$ th transfer, trapdoor information $Q^{\prime}$ and updated buyer's state information $D_{i}^{\prime}$.

- A request verification algorithm POTVerReq $\left(p k_{\mathcal{S}}, D_{i-1}\right.$, $Q)$ that, on input the public key $p k_{\mathcal{S}}$, seller's state information $D_{i-1}$ and request $Q$, verifies the request and outputs either accept or reject.

- An algorithm POTResp $\left(s k_{\mathcal{S}}, p k_{\mathcal{S}}, Q\right)$ that, on input secret key $s k_{\mathcal{S}}$, public key $p k_{\mathcal{S}}$ and request $Q$, outputs a response $R$ and updated seller's state information $D_{i}$.

${ }^{1}$ If the encrypted watermarked messages do not belong to the message space of the POT scheme used, its message space can be modified. For example, in the POT scheme in [29], a ciphertext of message $m$ consists of elements $(A, B)$, where $B=e(g, A) \oplus m$ for a bilinear map $e: \mathbb{G} \times \mathbb{G} \rightarrow \mathbb{G}_{t}$ and a generator $g$ of $\mathbb{G}$. A hash function $H$ can be used to map $e(g, A)$ to the key space of a secure symmetric key encryption scheme. The message is then encrypted via the symmetric key encryption scheme utilizing $H(e(g, A))$ as key. 
- A response verification algorithm $\operatorname{POTVerResp}\left(p k_{\mathcal{S}}, R\right)$ that, on input the public key $p k_{\mathcal{S}}$ and the response $R$, verifies the response and outputs either accept or reject.

- An algorithm POTComplete $\left(T, R, Q^{\prime}\right)$ that, on input the ciphertexts $T$, the response $R$ and trapdoor information $Q^{\prime}$, outputs the message $m_{\tau}$.

\section{Construction}

In the following, (JKeygen, JEnc, JDec) and (BKeygen, $\mathrm{BEnc}, \mathrm{BDec}$ ) stand for the algorithms for key generation, encryption and decryption of the public key encryption schemes used by $\mathcal{J}$ and $\mathcal{B}$ respectively. They are described in Section III. Additionally, (Keygen, Sign, VerifySig) are the algorithms for key generation, signing and signature verification of the signature scheme used by $\mathcal{B}$.

In the setup phase, each buyer $\mathcal{B}_{i}$ runs Keygen to obtain a key pair $\left(s k_{i}, p k_{i}\right)$ and registers $p k_{i}$ at $\mathcal{F}_{\text {REG }}$. The judge $\mathcal{J}$ runs his key generation algorithm JKeygen in order to generate a key pair $\left(s k_{\mathcal{J}}, p k_{\mathcal{J}}\right)$ and registers $p k_{\mathcal{J}}$ at $\mathcal{F}_{\text {REG }}$. Every party can retrieve public keys of other parties by querying $\mathcal{F}_{\text {REG }}$. The seller $\mathcal{S}$ executes the watermarking setup algorithm WATsetup to obtain a secret watermarking key $s w k$. The following phases are depicted in Figure 1 and described below.

\section{Protocol PBSW}

- Initialization. When $\mathcal{S}$ is activated with the message (init, $m_{1}, p_{1}, \ldots, m_{N}, p_{N}$ ) and each buyer $\mathcal{B}_{i}$ is activated with (deposit, $\left.a c_{i}\right), \mathcal{B}_{i}$ and $\mathcal{S}$ run algorithms $\operatorname{SetupB}\left(1^{\kappa}, s k_{i}, p k_{\mathcal{J}}\right)$ and $\operatorname{SetupV}\left(p k_{i}, p k_{\mathcal{J}}\right.$, $\left.s w k, m_{1}, \ldots, m_{N}\right)$ respectively. As results, $\mathcal{B}$ obtains a key pair $\left(s k_{\mathcal{B}}{ }^{\prime}, p k_{\mathcal{B}}{ }^{\prime}\right) . \mathcal{S}$ obtains encrypted watermarked messages $\left(c t_{1}, \ldots, c t_{j}\right)$ and state information info that allows the identification of $\mathcal{B}_{i}$ when she releases a pirated copy related with $\left(c t_{1}\right.$, $\left.\ldots, c t_{j}\right)$. Then $\mathcal{S}$ runs POTInitS $\left(1^{\kappa}, c t_{1}, p_{1}, \ldots\right.$, $\left.c t_{N}, p_{N}, D_{\max }\right)$ to obtain a secret key $s k_{\mathcal{S}}$ and a public key $p k_{\mathcal{S}}$ of the POT scheme, and a set of $N$ ciphertexts $T . \mathcal{S}$ sends $\left(p k_{\mathcal{S}}, T\right)$ to $\mathcal{B} . \mathcal{B}$ runs $\left(P, D_{0}^{\prime}\right) \leftarrow \operatorname{POTInitB}\left(1^{\kappa}, p k_{\mathcal{S}}, T, a c_{0}\right)$ and aborts if the output is reject. Otherwise, $\mathcal{B}$ sends the payment message $(P)$ to $\mathcal{S}$ and pays an amount of $a c_{0}$ through an arbitrary payment channel. $\mathcal{S}$ runs $\left(D_{0}, a c_{0}\right) \leftarrow \operatorname{POTGetDep}\left(s k_{\mathcal{S}}, P, D_{\max }\right)$ and checks that $a c_{0}$ corresponds to the amount of money received. $\mathcal{S}$ adds $\left(\mathcal{B}_{i}\right.$, info $)$ to a buyer's database $\mathrm{DB}_{\text {info }}$, stores state information $U_{0}=\left(s k_{\mathcal{S}}, p k_{\mathcal{S}}\right.$, $T, D_{0}$ ), and outputs (deposit, $\left.a c_{0}\right) . \mathcal{B}_{i}$ stores state information $U_{0}^{\prime}=\left(p k_{\mathcal{S}}, T, D_{0}^{\prime}\right)$.

- Purchase. When $\mathcal{B}_{i}$ is activated with (request, $\tau_{i}$ ), $\mathcal{B}_{i}$ runs POTReq $\left(p k_{\mathcal{S}}, T, D_{i-1}^{\prime}, \tau_{i}\right)$ to get a request $Q$, a trapdoor $Q^{\prime}$ and private state $D_{i}^{\prime}$. $\mathcal{B}$ sends $(Q)$ and stores $\left(Q^{\prime}, D_{i}^{\prime}\right) . \mathcal{S}$ runs POTVerReq $\left(p k_{\mathcal{S}}\right.$, $\left.D_{i-1}, Q\right)$ and ignores the request if the output is reject. Otherwise $\mathcal{S}$ runs $\operatorname{POTResp}\left(s k_{\mathcal{S}}, p k_{\mathcal{S}}, Q\right)$ to obtain a response $R$ and state $D_{i}$, and sends
$(R)$ to $\mathcal{B}$. $\mathcal{B}$ runs $\operatorname{POTVerResp}\left(p k_{\mathcal{S}}, R\right)$ and outputs (response, $\perp$ ) if the output is reject. Otherwise $\mathcal{B}$ runs POTComplete $\left(T, R, Q^{\prime}\right)$ to obtain $c t_{\tau_{i}}$ and runs $\operatorname{Dec}\left(s k_{\mathcal{B}}{ }^{\prime}, c t_{\tau_{i}}\right)$ to get $y_{\tau_{i}}$, i.e., the message $m_{\tau_{i}}$ watermarked. $\mathcal{S}$ stores state information $U_{i}=\left(s k_{\mathcal{S}}\right.$, $\left.p k_{\mathcal{S}}, T, D_{i}\right)$, and $\mathcal{B}$ stores state information $U_{i}^{\prime}=$ $\left(p k_{\mathcal{S}}, T, D_{i}^{\prime}\right)$ and outputs (response, $\left.y_{\tau_{i}}\right)$.

- Arbitration. When $\mathcal{S}$ is activated with (detect, $y$ ), $\mathcal{S}$ runs Detect $\left(s w k, y, \mathrm{DB}_{\text {info }}\right)$ to obtain a framing message $F$ and sends $(F)$ to $\mathcal{J}$. $\mathcal{J}$ retrieves the public key $p k_{i}$ of the buyer $\mathcal{B}_{i}$ in $F$ and runs $\operatorname{Check}\left(p k_{i}, F, s k_{\mathcal{J}}\right)$ to obtain a bit $b$. If $b=0$, $\mathcal{J}$ sends (not guilty) to $\mathcal{S}$ and outputs (detresp, not guilty). Otherwise $\mathcal{J}$ sends (guilty) to $\mathcal{S}$ and outputs (detresp, guilty).

- SetupB $\left(1^{\kappa}, s k_{i}, p k_{\mathcal{J}}\right)$. Run BKeygen $\left(1^{\kappa}\right)$ to obtain a key pair $\left(s k_{\mathcal{B}}{ }^{\prime}, p k_{\mathcal{B}}{ }^{\prime}\right)$. Run JEnc $\left(p k_{\mathcal{J}}, s k_{\mathcal{B}}{ }^{\prime}\right)$ to get an encryption $C$ of $s k_{\mathcal{B}}{ }^{\prime}$. Pick a random string $W_{\mathcal{B}} \leftarrow\{0,1\}^{l_{2}}$ and, for $i=1$ to $l_{2}$, run $c_{i}=\operatorname{BEnc}\left(p k_{\mathcal{B}^{\prime}}{ }^{\prime}, W_{\mathcal{B} i}\right)$ to encrypt bitwise $W_{\mathcal{B}}$. Set a message $m=\left(p k_{\mathcal{B}}{ }^{\prime},\left(c_{i}\right)_{i=1}^{l_{2}}, C\right)$ and run $\operatorname{Sign}\left(s k_{i}, m\right)$ to compute a signature $s_{m}$. (If $m$ does not belong to the message space of the group signature scheme, use a collision-resistant hash function $H$ to compute a hash $H(m)$ that belongs to the message space and sign $H(m)$.) Send $\left(m, s_{m}\right)$ to $\mathcal{S}$. As the prover, engage with $\mathcal{S}$ in the following interactive zero-knowledge proofs of knowledge: a proof $\pi_{1}=\operatorname{PK}\left\{\left(s k_{\mathcal{B}}{ }^{\prime}\right):\left(p k_{\mathcal{B}}{ }^{\prime}, s k_{\mathcal{B}}{ }^{\prime}\right) \leftarrow\right.$ $\left.\operatorname{BKeygen}\left(1^{k}\right) \wedge C \leftarrow \operatorname{JEnc}\left(p k_{\mathcal{J}}, s k_{\mathcal{B}}{ }^{\prime}\right)\right\}$ that $\left(p k_{\mathcal{B}}{ }^{\prime}, s k_{\mathcal{B}}{ }^{\prime}\right)$ are correctly setup and that $C$ is an encryption of $s k_{\mathcal{B}}{ }^{\prime}$ with $p k_{\mathcal{J}}$; for $i=1$ to $l_{2}$, a proof $\pi_{2 i}=\operatorname{PK}\left\{\left(W_{\mathcal{B}_{i}}\right)\right.$ : $\left.c_{i} \leftarrow \operatorname{BEnc}\left(p k_{\mathcal{B}^{\prime}}{ }^{\prime}, W_{\mathcal{B}_{i}}\right) \wedge W_{\mathcal{B}_{i}} \in\{0,1\}\right\}$ that each $c_{i}$ encrypts a bit. Output a key pair $\left(s k_{\mathcal{B}^{\prime}}{ }^{\prime}, p k_{\mathcal{B}}{ }^{\prime}\right)$.

- SetupV $\left(p k_{i}, p k_{\mathcal{J}}, s w k, m_{1}, \ldots, m_{N}\right)$. Receive tuple $(m$, $\left.s_{m}\right)$ and parse the message $m$ as $\left(p k_{\mathcal{B}}{ }^{\prime},\left(c_{i}\right)_{i=1}^{l_{2}}, C\right)$. Run VerifySig $\left(p k_{i}, m, s_{m}\right)$ and abort if the output is reject. As the verifier, engage in the execution of the interactive proofs $\pi_{1}$ and, for $i=1$ to $l_{2}, \pi_{2 i}$, and abort if any of them is not correct. For $j=1$ to $N$, pick random $W_{\mathcal{S}_{j}} \leftarrow\{0,1\}^{l_{2}}$ and, for $i=1$ to $l_{2}$, run BEnc $\left(p k_{\mathcal{B}}{ }^{\prime}, W_{\mathcal{S}_{j i}} \oplus W_{\mathcal{B}_{i}}\right)$. Pick random unique $\phi_{j} \leftarrow\{0,1\}^{l_{1}}$ and, for $i=1$ to $l_{1}$, encrypt $\operatorname{BEnc}\left(p k_{\mathcal{B}}{ }^{\prime}, \phi_{j i}\right)$. Set the watermark to be embedded as $w_{j}=\phi_{j} \|\left(W_{\mathcal{S}_{j}} \oplus W_{\mathcal{B}}\right)$, and let its bitwise encryption be $\operatorname{BEnc}\left(p k_{\mathcal{B}}{ }^{\prime}, w_{j}\right)$. Perform the watermark embedding operation WATemb $\left(s w k, m_{j}\right.$, $\left.\operatorname{BEnc}\left(p k_{\mathcal{B}}{ }^{\prime}, w_{j}\right)\right)$ in the encrypted domain to obtain an encrypted watermarked message $c t_{j}=\operatorname{BEnc}\left(p k_{\mathcal{B}}{ }^{\prime}, y_{j}\right)$. Output encrypted watermarked messages $\left(c t_{1}, \ldots, c t_{N}\right)$ and state information info $=\left\{m, s_{m},\left(\phi_{j}, W_{\mathcal{S}_{j}}\right)_{j=1}^{N}\right\}$.

- Detect $\left(s w k, y, \mathrm{DB}_{i n f o}\right)$. Execute the watermark detection algorithm WATdet $(s w k, y)$ to obtain the watermark $w=$ $\phi \| x$, parse the entry $\left(\mathcal{B}_{i}, \phi, W_{\mathcal{S}}, m, s_{m}\right)$ of table $\mathrm{DB}_{\text {info }}$, compute $W_{\mathcal{B}}=W_{\mathcal{S}} \oplus x$ and output $F=\left(W_{\mathcal{B}}, m, s_{m}\right.$, $\left.\mathcal{B}_{i}\right)$.

- Check $\left(p k_{i}, F, s k_{\mathcal{J}}\right)$. Parse $F$ as $\left(W_{\mathcal{B}}, m, s_{m}, \mathcal{B}_{i}\right)$ and $m$ as $\left(p k_{\mathcal{B}},\left(c_{i}\right)_{i=1}^{l_{2}}, C\right)$. Run VerifySig $\left(p k_{i}, m, s_{m}\right)$ and 


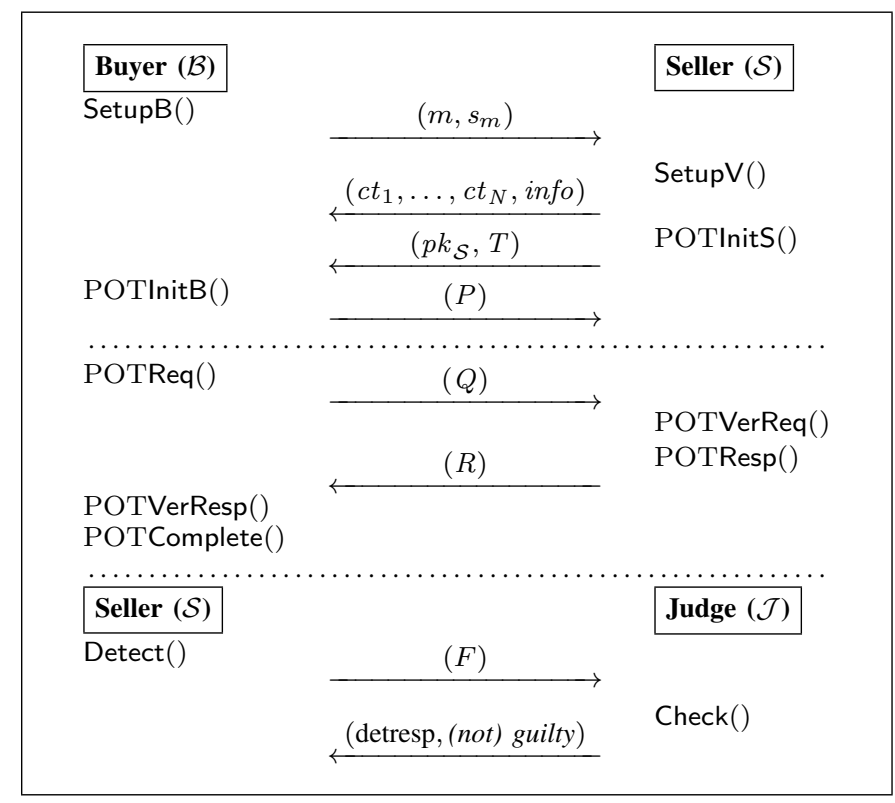

Fig. 1. Phases of PBSW protocol: initialization (top), purchase (middle), arbitration (bottom).

abort if the output is 0 . Decrypt $\operatorname{JDec}\left(s k_{\mathcal{J}}, C\right)$ to obtain $s k_{\mathcal{B}}{ }^{\prime}$. For $i=1$ to $l_{2}$, decrypt $\operatorname{BDec}\left(s k_{\mathcal{B}}{ }^{\prime}, c_{i}\right)$ to obtain $W_{\mathcal{B}}{ }^{\prime}$. Check whether $W_{\mathcal{B}}{ }^{\prime}=W_{\mathcal{B}}$. If it is the case, output $b=1$ and otherwise $b=0$.

\section{SECURITy ANALYSIS}

Theorem 1. This $\mathrm{PBSW}$ scheme securely realizes $\mathcal{F}_{\mathrm{PCP}}$.

In order to prove this theorem, we need to build a simulator $\mathcal{E}$ that invokes a copy of adversary $\mathcal{A}$ and interacts with $\mathcal{F}_{\mathrm{PCP}}$ and environment $\mathcal{Z}$ in such a way that ensembles $\mathrm{IDEAL}_{\mathcal{F}_{\mathrm{PCP}}, \mathcal{E}, \mathcal{Z}}$ and $\mathrm{REAL}_{\mathrm{PCP}, \mathcal{A}, \mathcal{Z}}$ are computationally indistinguishable.

We prove security of our protocol under the assumption that it is instantiated with a secure POT scheme, i.e., one that realizes functionality $\mathcal{F}_{\text {POT }}$ described in Section II. The security of the POT scheme implies the existence of a simulator $\mathcal{E}_{\mathrm{POT}}$ that interacts with $\mathcal{F}_{\mathrm{POT}}$ and $\mathcal{Z}$ in such a way that $\mathcal{Z}$ cannot distinguish the ideal world from the real world. Our simulator $\mathcal{E}$ employs simulator $\mathcal{E}_{\mathrm{POT}}$.

We analyze formally the security of our scheme when the seller and a subset of buyers are corrupted, and when (a subset of) buyers are corrupted. We denote by $\nu$ a negligible function.

\section{A. Security Analysis When Seller Is Corrupted}

Claim 1. When the seller and a subset of the buyers are corrupted, the ensembles $\operatorname{IDEAL}_{\mathcal{F}_{\mathrm{PCP}}, \mathcal{E}, \mathcal{Z}}$ and $\mathrm{REAL}_{\mathrm{PCP}, \mathcal{A}, \mathcal{Z}}$ are computationally indistinguishable under the security of the POT scheme, the zero-knowledge property of the proofs of knowledge, the IND-CPA security of encryption schemes (BKeygen, BEnc, BDec) and (JKeygen, JEnc, JDec), and the unforgeability of the signature scheme (Keygen, Sign, VerifySig).
Proof: We show by means of a series of hybrid games that the environment $\mathcal{Z}$ cannot distinguish between the real ex-

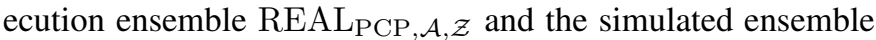
IDEAL $\mathcal{F}_{\mathcal{F}_{\mathrm{PCP}}, \mathcal{E}, \mathcal{Z}}$ with non-negligible probability. We denote by $\operatorname{Pr}[$ Game $i]$ the probability that $\mathcal{Z}$ distinguishes between the ensemble of Game $i$ and that of the real execution.

- Game 0: This game corresponds to the execution of the real-world protocol with a subset of honest buyers and an honest $\mathcal{J}$. Thus $\operatorname{Pr}[$ Game 0$]=0$.

- Game 1: This game proceeds as Game 0, except that Game 1 aborts if $\mathcal{A}$ sends a message-signature pair $\left(m, s_{m}\right)$ correct according to algorithm $\operatorname{Verify} \operatorname{Sig}\left(p k_{i}, m\right.$, $\left.s_{m}\right)$, where $p k_{i}$ is the public key of an honest buyer and such that $\mathcal{A}$ did not obtain before a signature of message $m$ for public key $p k_{i}$. The probability that Game 1 aborts is bounded by the following lemma:

Lemma 1. Under the existential unforgeability of the signature scheme (Keygen, Sign, VerifySig), $\mid \operatorname{Pr}$ [Game 1] $\operatorname{Pr}[$ Game 0$] \mid=\nu_{1}$.

Proof: We construct an algorithm $\mathcal{T}$ that, if there exists an adversary $\mathcal{A}$ that makes Game 1 abort with nonnegligible probability $\epsilon$, breaks the existential unforgeability of the signature scheme with non-negligible probability $\epsilon / n$, where $n$ denotes the number of honest buyers. Existential unforgeability is formally defined in [21] as a game between a challenger $\mathcal{C}$ and an adversary. First, $\mathcal{C}$ gives to the adversary a public key $p k$ and access to a signing oracle $\mathrm{O}(s k, \cdot)$. Eventually, $\mathcal{A}$ outputs a message signature pair $\left(m, s_{m}\right)$, and wins the game if VerifySig $\left(p k, m, s_{m}\right)$ outputs accept and $\mathrm{O}(s k, \cdot)$ was not queried on input $m$.

Algorithm $\mathcal{T}$ operates as follows. First, $\mathcal{T}$ receives $p k$ from $\mathcal{C}$. $\mathcal{T}$ chooses an honest buyer $\mathcal{B}_{i}$, and, when $\mathcal{A}$ queries the public key of $\mathcal{B}_{i}, \mathcal{T}$ sends $p k$. To simulate a purchase request from $\mathcal{B}_{i}, \mathcal{T}$ queries $\mathrm{O}(s k, \cdot)$ on input the purchase message $m$ to obtain $s_{m}$ and sends $\left(m, s_{m}\right)$ to $\mathcal{A}$. Eventually, $\mathcal{A}$ sends a pair $\left(m^{\prime}, s_{m^{\prime}}\right)$ such that Game 1 aborts. If $s_{m^{\prime}}$ is not associated with public key $p k, \mathcal{T}$ fails. Otherwise, $\mathcal{T}$ submits $\left(m^{\prime}, s_{m^{\prime}}\right)$ to $\mathcal{C}$.

- Game 2: This game proceeds as Game 1, except that the proofs $\pi_{1}=\operatorname{PK}\left\{\left(s k_{\mathcal{B}}{ }^{\prime}\right):\left(p k_{\mathcal{B}}{ }^{\prime}, s k_{\mathcal{B}}{ }^{\prime}\right) \leftarrow\right.$ BKeygen $\left(1^{k}\right) \wedge$ $\left.C \leftarrow \operatorname{JEnc}\left(p k_{\mathcal{J}}, s k_{\mathcal{B}^{\prime}}{ }^{\prime}\right)\right\}$ and $\left\{\pi_{2 i}=\operatorname{PK}\left\{\left(W_{\mathcal{B}_{i}}\right): c_{i} \leftarrow\right.\right.$ $\left.\left.\operatorname{BEnc}\left(p k_{\mathcal{B}^{\prime}}{ }^{\prime}, W_{\mathcal{B}_{i}}\right) \wedge W_{\mathcal{B}_{i}} \in\{0,1\}\right\}\right\}_{i=1}^{l_{2}}$ are replaced by simulated proofs. Under the assumption that the proof system is zero-knowledge, $\mid \operatorname{Pr}[$ Game 2] $-\operatorname{Pr}[$ Game 1] $\mid=$ $\nu_{2}$.

- Game 3: This game proceeds as Game 2, except that the ciphertext $C=\operatorname{JEnc}\left(p k_{\mathcal{J}}, s k_{\mathcal{B}}{ }^{\prime}\right)$ included in the initialization message of buyers is replaced by a ciphertext that encrypts a random message. At this point, the proof of knowledge $\pi_{1}=\operatorname{PK}\left\{\left(s k_{\mathcal{B}}{ }^{\prime}\right):\left(p k_{\mathcal{B}}{ }^{\prime}, s k_{\mathcal{B}}{ }^{\prime}\right) \leftarrow\right.$ $\left.\operatorname{BKeygen}\left(1^{k}\right) \wedge C \leftarrow \operatorname{JEnc}\left(p k_{\mathcal{J}}, s k_{\mathcal{B}}{ }^{\prime}\right)\right\}$ is a simulated proof of a false statement. The probability that $\mathcal{Z}$ distinguishes between Game 3 and Game 2 is bounded by the following lemma:

Lemma 2. Under the IND-CPA security of the encryption scheme that consists of algorithms (JKeygen, JEnc, 
JDec), $\mid \operatorname{Pr}[$ Game 3$]-\operatorname{Pr}[$ Game 2] $]=\nu_{3}$.

Proof: We construct an algorithm $\mathcal{T}$ that, given an environment $\mathcal{Z}$ that distinguishes Game 3 and Game 2 with non-negligible probability, breaks the IND-CPA security of the encryption scheme with non-negligible probability. Chosen plaintext security is formally defined through a game between a challenger $\mathcal{C}$ and an adversary [22]. First, $\mathcal{C}$ provides the adversary with a public key $p k$. The adversary sends two messages $m_{0}$ and $m_{1}$. $\mathcal{C}$ flips a coin $b$ and sends $C=\operatorname{Enc}\left(p k, m_{b}\right)$ to the adversary. Finally, the adversary sends his guess $b^{\prime}$ and wins if $\left|\operatorname{Pr}\left[b=b^{\prime}\right]-\frac{1}{2}\right|$ is non-negligible.

Let $n$ be the number of honest buyers. We consider a sequence of hybrid games, where, in game- $j$, ciphertext $C$ is replaced by the encryption of a random message in the initialization messages of buyers $\mathcal{B}_{1}, \ldots, \mathcal{B}_{j}$, while the remaining requests remain unchanged. Clearly, game0 corresponds to Game 2 and game- $n$ corresponds to Game 3. If $\mathcal{Z}$ distinguishes Game 3 and Game 2 with non-negligible probability $\epsilon$, there must be an index $j$ such that $\mathcal{Z}$ distinguishes game- $j$ from game- $(j+1)$ with non-negligible probability $\epsilon / n$.

Our algorithm $\mathcal{T}$ operates as follows. First, $\mathcal{T}$ receives the public key $p k$ from $\mathcal{C}$. When $\mathcal{A}$ requests the public key of $\mathcal{J}, \mathcal{T}$ sends $p k$. Initialization messages of buyers $\mathcal{B}_{1}, \ldots, \mathcal{B}_{j}$ are computed following algorithm SetupB, except that $C$ is replaced by the encryption of a random value and $\pi_{1}$ and $\left\{\pi_{2 i}\right\}_{i=1}^{l_{2}}$ by simulated proofs. For $\mathcal{B}_{j+2}, \ldots, \mathcal{B}_{n}$, initialization messages are computed following algorithm SetupB. For $\mathcal{B}_{j+1}, \mathcal{T}$ computes BKeygen $\left(1^{\kappa}\right)$ to obtain a key pair $\left(s k_{\mathcal{B}}{ }^{\prime}, p k_{\mathcal{B}}{ }^{\prime}\right)$, picks random $m$ and submits $\left(s k_{\mathcal{B}^{\prime}}{ }^{\prime}, m\right)$ to $\mathcal{C}$. $\mathcal{C}$ flips a coin $b$ and returns $C=\operatorname{JEnc}\left(p k, m_{b}\right)$, and $\mathcal{T}$ uses $C$ to compute the request. If $b=0$, the distribution corresponds to game- $j$, and, if $b=1$, it corresponds to game- $j+1$. $\mathcal{Z}$ outputs a bit $b^{\prime}$, which is forwarded by $\mathcal{T}$ to $\mathcal{C}$.

- Game 4: This game proceeds as Game 3, except that Game 4 aborts upon receiving an arbitration request $\left(W_{\mathcal{B}}, m, s_{m}, \mathcal{B}_{i}\right)$ where $\left(m, s_{m}\right)$ was previously sent to $\mathcal{A}, s_{m}$ is associated with the public key of an honest buyer $\mathcal{B}_{i}$ and $W_{\mathcal{B}}$ was the buyer's watermark sent by $\mathcal{B}_{i}$ in the initialization phase. The probability that $\mathcal{Z}$ distinguishes between Game 4 and Game 3 is bounded by the following lemma:

Lemma 3. Under the IND-CPA security of the encryption scheme that consists of algorithms (BKeygen, BEnc, BDec), $\mid \operatorname{Pr}[$ Game 4$]-\operatorname{Pr}\left[\right.$ Game 3] $\mid=\nu_{4}$.

Proof: Let $n$ be the number of honest buyers. We construct an algorithm $\mathcal{T}$ that, given an adversary $\mathcal{A}$ that makes Game 4 abort with non-negligible probability, breaks the chosen plaintext security of the encryption scheme with non-negligible probability $\epsilon / n$.

Algorithm $\mathcal{T}$ operates as follows. First, $\mathcal{T}$ receives a public key $p k_{\mathcal{B}}{ }^{\prime}$ from $\mathcal{C}$. To compute the initialization message $m$ of $\mathcal{B}_{1}, \mathcal{T}$ follows Game 3 and uses $p k_{\mathcal{B}}{ }^{\prime}$ to encrypt bitwise $W_{\mathcal{B}}$ and obtain $\left(c_{i}\right)_{i=1}^{l_{2}}$. To encrypt the first $l_{2}-1$ bits, $\mathcal{T}$ encrypts bitwise $W_{\mathcal{B}}$ using
$c_{i}=\operatorname{BEnc}\left(p k_{\mathcal{B}}{ }^{\prime}, m_{i}\right)$. To encrypt the last bit, $\mathcal{T}$ sends $(0,1)$ to $\mathcal{C}$ and receives back a ciphertext $c$, which is used to complete the bitwise encryption. To compute the initialization message of buyers $\mathcal{B}_{2}, \ldots, \mathcal{B}_{n}, \mathcal{T}$ follows Game 3. Eventually $\mathcal{A}$ sends an arbitration message $\left(W_{\mathcal{B}}, m, s_{m}, \mathcal{B}_{i}\right)$ that makes Game 4 abort. If $\mathcal{B}_{i} \neq \mathcal{B}_{1}$, $\mathcal{T}$ fails. Otherwise, if the last bit of $W_{\mathcal{B}}$ is $0, \mathcal{T}$ sends $b^{\prime}=0$ to $\mathcal{C}$, and otherwise $b^{\prime}=1$ to $\mathcal{C}$.

- Game 5: This game proceeds as Game 4, except that Game 5 performs all the changes described in $\mathcal{E}_{\text {POT }}$ for the case in which sender and a subset of buyers are corrupted. Under the security of the POT scheme, we have that $\mid \operatorname{Pr}\left[\right.$ Game 5] $-\operatorname{Pr}\left[\right.$ Game 4] $\mid=\nu_{5}$.

$\mathcal{E}$ performs all the changes described in Game 5, and forwards and receives messages from $\mathcal{F}_{\mathrm{PCP}}$ as described in our simulation below:

- Setup. When $\mathcal{A}$ sends a request (retrieve, $\mathcal{J}$ ), $\mathcal{E}$ runs JKeygen in order to generate a key pair $\left(s k_{\mathcal{J}}, p k_{\mathcal{J}}\right)$ and sends (retrieve, $\mathcal{J}, p k_{\mathcal{J}}$ ) to $\mathcal{A}$. Similarly, when $\mathcal{A}$ sends a request (retrieve, $\mathcal{B}_{i}$ ), where $\mathcal{B}_{i}$ is an uncorrupted buyer, $\mathcal{E}$ runs Keygen to generate $\left(s k_{i}, p k_{i}\right)$ and returns (retrieve, $\mathcal{J}, p k_{i}$ ) to $\mathcal{A}$. When $\mathcal{A}$ sends (register, $p k_{j}$ ) to register the public key $p k_{j}$ of a corrupted buyer $\mathcal{B}_{j}, \mathcal{E}$ stores $p k_{j}$.

- Initialization. $\mathcal{E}$ follows Game 5 to compute the initialization message $\left(m, s_{m}\right)$ of each honest buyer $\mathcal{B}_{i}$ using $p k_{\mathcal{B}_{i}}{ }^{\prime}$ as encryption public key, and runs the subsequent simulated zero-knowledge proofs of knowledge $\pi_{1}$ and $\left\{\pi_{2 i}\right\}_{i=1}^{l_{2}}$ with $\mathcal{A}$. Upon receiving $\left(p k_{\mathcal{S}}, T\right)$ from $\mathcal{A}, \mathcal{E}$ runs $\mathcal{E}_{\mathrm{POT}}$ on input $\left(p k_{\mathcal{S}}, T\right)$ and obtains (init, $c t_{1}, p_{1}$, $\left.\ldots, c t_{N}, p_{N}\right)$. For $j=1$ to $N, \mathcal{E}$ runs $\operatorname{Dec}\left(s k_{\mathcal{B}_{i}}^{\prime}, c t_{j}\right)$ to obtain $y_{i j}$. After obtaining $y_{i 1}, \ldots, y_{i N}$ for all buyers $\mathcal{B}_{i}, \mathcal{E}$ sends (init, $\left.\left\{\mathcal{B}_{i}, y_{i 1}, \ldots, y_{i N}\right\}_{i=1}^{n}, p_{1}, \ldots, p_{N}\right)$ to $\mathcal{F}_{\mathrm{PCP}}$. Upon receiving (deposit, $\mathcal{B}_{i}, a c_{i}$ ) from $\mathcal{F}_{\mathrm{PCP}}$, $\mathcal{B}_{i}$ runs $\mathcal{E}_{\mathrm{POT}}$ on input (deposit, $\mathcal{B}_{i}, a c_{i}$ ) and forwards messages between $\mathcal{E}_{\mathrm{POT}}$ and $\mathcal{A}$.

- Purchase. Upon receiving (request, $\mathcal{B}_{i}$ ) from $\mathcal{F}_{\mathrm{PCP}}, \mathcal{E}$ runs $\mathcal{E}_{\mathrm{POT}}$ on input (request, $\mathcal{B}_{i}$ ) and forwards messages between $\mathcal{E}_{\mathrm{POT}}$ and $\mathcal{A}$. When $\mathcal{E}_{\mathrm{POT}}$ returns (response, $b$ ), $\mathcal{E}$ sends (response, $b$ ) to $\mathcal{F}_{\mathrm{PCP}}$.

- Release. Upon receiving a pirated copy $y^{\prime}$ from $\mathcal{A}, \mathcal{E}$ sends (release, $y^{\prime}$ ) to $\mathcal{F}_{\mathrm{PCP}}$ and stores $y^{\prime}$ in a table $T_{\text {rel }}$ of released copies.

- Arbitration. Upon receiving a framing message $(F)$ from $\mathcal{A}, \mathcal{E}$ aborts if any of the conditions described up to Game 5 is fulfilled. (We showed that $\mathcal{E}$ aborts with negligible probability.) Those conditions prevent $\mathcal{A}$ from framing honest buyers, who by assumption do not release pirated copies. If $\mathcal{E}$ does not abort, $\mathcal{E}$ picks any pirated copy in $T_{r e l}$ and sends (detect, $y$ ) to $\mathcal{F}_{\mathrm{PCP}}$. Upon receiving (detresp, $\mathcal{E}$, guilty), $\mathcal{E}$ sends (guilty) to $\mathcal{A}$.

The distribution produced in Game 5 is identical to that of our simulation. By summation we have that $\mid \operatorname{Pr}\left[\right.$ Game 5] $\leq \nu_{6}$. 


\section{B. Security Analysis When Buyers Are Corrupted}

Claim 2. When only the buyers are corrupted, the ensembles $\mathrm{IDEAL}_{\mathcal{F}_{\mathrm{PCP}}, \mathcal{E}, \mathcal{Z}}$ and $\mathrm{REAL}_{\mathrm{PCP}, \mathcal{A}, \mathcal{Z}}$ are computationally indistinguishable under the security of the POT scheme, the unforgeability of the signature scheme (Keygen, Sign, VerifySig) and the collusion resistance of the watermarking scheme.

Proof: We show by means of a series of hybrid games that the environment $\mathcal{Z}$ cannot distinguish between the real execution ensemble $\mathrm{REAL}_{\mathrm{PCP}, \mathcal{A}, \mathcal{Z}}$ and the simulated ensemble $\mathrm{IDEAL}_{\mathcal{F}_{\mathrm{PCP}}, \mathcal{E}, \mathcal{Z}}$ with non-negligible probability.

- Game 0: This game corresponds to the execution of the real-world protocol with honest $\mathcal{S}$ and $\mathcal{J}$. Therefore, $\operatorname{Pr}[$ Game 0$]=0$.

- Game 1 : This game operates as Game 0, except that the string $w=\phi \|\left(W_{\mathcal{S}} \oplus W_{\mathcal{B}}\right)$ that is used to compute the watermark embedding is replaced by a random string. Since the strings $\phi$ and $W_{\mathcal{S}}$ are picked at random by the honest seller, $w$ is a random string that leaks no information on $W_{\mathcal{B}}$. Therefore, $\mid \operatorname{Pr}[$ Game 1] $-\operatorname{Pr}[$ Game 0] $\mid=0$.

- Game 2 : This game operates as Game 1, except that Game 2 aborts if $\mathcal{A}$ releases a watermarked content $y$ whose watermark $w$ does not equal that of any of the watermarked contents previously received by $\mathcal{A}$. The probability that Game 2 aborts is bounded by the following lemma:

Lemma 4. Under the assumption that the watermarking scheme is collusion resistant, $\mid \operatorname{Pr}[$ Game 2] $\operatorname{Pr}\left[\right.$ Game 1] $\mid=\nu_{1}$.

Proof: We construct an algorithm $\mathcal{T}$ that, given an adversary $\mathcal{A}$ that makes Game 2 abort with nonnegligible probability, breaks the collusion resistant property of the watermarking scheme with non-negligible probability. $\mathcal{T}$ interacts with the challenger $\mathcal{C}$ of the collusion resistant game described in Definition 1. First, $\mathcal{T}$ receives the challenge $\left(y_{1}, \ldots, y_{l}\right)$ from $\mathcal{C} . \mathcal{T}$ computes the public key $p k_{\mathcal{J}}$ of $\mathcal{J}$ and sends $p k_{\mathcal{J}}$ to $\mathcal{A}$ upon request. $\mathcal{T}$ employs $\left(y_{1}, \ldots, y_{l}\right)$ as the watermarked copy of messages $m_{1}$. (We assume that $l$ is larger than the number of buyers $n$.) For other items, the watermarked copies are computed as usual. Eventually, $\mathcal{A}$ releases a pirated copy $y^{\prime}$ that corresponds to $m_{1}$ whose watermark does not equal any of the watermarks embedded in $\left(y_{1}, \ldots, y_{l}\right) . \mathcal{T}$ forwards $y^{\prime}$ to $\mathcal{C}$.

- Game 3: This game proceeds as Game 2, except that Game 3 performs all the changes described in $\mathcal{E}_{\mathrm{POT}}$ for the case in which the buyers are corrupted. Under the security of the POT scheme, we have that $\mid \operatorname{Pr}$ [Game 3]$\operatorname{Pr}\left[\right.$ Game 2] $=\nu_{2}$.

$\mathcal{E}$ performs all the changes described in Game 3, and forwards and receives messages from $\mathcal{F}_{\mathrm{PCP}}$ as described in our simulation below:

- Setup. When $\mathcal{A}$ sends a request (retrieve, $\mathcal{J}), \mathcal{E}$ runs JKeygen in order to generate a key pair $\left(s k_{\mathcal{J}}, p k_{\mathcal{J}}\right)$ and sends (retrieve, $\mathcal{J}, p k_{\mathcal{J}}$ ) to $\mathcal{A}$. When $\mathcal{A}$ sends (register, $p k_{j}$ ) to register the public key $p k_{j}$ of a corrupted buyer $\mathcal{B}_{j}, \mathcal{E}$ stores $p k_{j}$.
- Initialization. $\mathcal{E}$ receives the message (init, $p_{1}, \ldots, p_{N}$ ) and stores it. Upon receiving $\left(m, s_{m}\right)$ from $\mathcal{A}$, where $s_{m}$ is associated with the public key of buyer $\mathcal{B}_{i}, \mathcal{E}$ checks whether VerifySig outputs accept. As verifier, $\mathcal{E}$ executes the proofs $\pi_{1}$ and, for $i=1$ to $l_{2}, \pi_{2 i}$, and ignores the request if any of them fails. Otherwise $\mathcal{E}$ stores the tuple $\left(\mathcal{B}_{i}, m, s_{m}\right)$. $\mathcal{E}$ runs $\mathcal{E}_{\mathrm{POT}}$ on input (init, $\left.p_{1}, \ldots, p_{N}\right)$ and forwards messages between $\mathcal{E}_{\mathrm{POT}}$ and $\mathcal{A}$. When $\mathcal{E}_{\mathrm{POT}}$ returns (deposit, $a c_{i}$ ), $\mathcal{E}$ sends (deposit, $a c_{i}$ ) to $\mathcal{F}_{\mathrm{PCP}}$.

- Purchase. Upon receiving a request $Q$ from $\mathcal{A}$ on behalf of $\mathcal{B}_{i}, \mathcal{E}$ runs $\mathcal{E}_{\mathrm{POT}}$ on input $Q$. $\mathcal{E}_{\mathrm{POT}}$ returns (request, $\tau$ ), and $\mathcal{E}$ sends (request, $\tau$ ) to $\mathcal{F}_{\mathrm{PCP}} . \mathcal{F}_{\mathrm{PCP}}$ returns (response, $\left.y_{i \tau}\right) . \mathcal{E}$ picks the tuple $\left(\mathcal{B}_{i}, m, s_{m}\right)$, parses $m$ as $\left(p k_{\mathcal{B}}{ }^{\prime},\left(c_{i}\right)_{i=1}^{l_{2}}, C\right)$ and encrypts $y_{i \tau}$ by running $c t_{i \tau}=\operatorname{Enc}\left(p k_{\mathcal{B}}{ }^{\prime}, y_{i \tau}\right)$. $\mathcal{E}$ runs $\mathcal{E}_{\mathrm{POT}}$ on input (response, $c t_{i \tau}$ ) and forwards messages between $\mathcal{E}_{\mathrm{POT}}$ and $\mathcal{A}$.

- Release. Upon receiving a pirated copy $y^{\prime}$ from $\mathcal{A}, \mathcal{E}$ sends (release, $y^{\prime}$ ) to $\mathcal{F}_{\mathrm{PCP}}$.

The distribution produced in Game 3 is identical to that of our simulation. By summation we have that $\mid \operatorname{Pr}\left[\right.$ Game 3] $\leq \nu_{3}$.

\section{FAIR PRIVACY-PRESERVING BSW PROTOCOL}

Recently, a transformation that takes as input a secure POT scheme and turns it into an optimistic fair POT scheme has been proposed [29]. This transformation requires a neutral third party, an adjudicator, who is only involved in case of dispute between a seller and a buyer (hence the protocol is called optimistic). Other fair e-commerce protocols that do not protect privacy also require the involvement of a third party [30].

The transformation is based on the use of verifiably encrypted signatures (VES) [31]. Roughly speaking, a VES is a signature encrypted under the public key of the adjudicator that can be publicly verified, i.e., the verifier can check that the ciphertext contains a valid signature without the secret key of the adjudicator.

The transformation works as follows. The buyer computes a VES $\omega$ on her purchase request $Q$, and sends $(Q, \omega)$ to the seller. Upon receiving a correct response from seller, the buyer reveals a valid signature on her request. This signature can be used by the seller to prove that the buyer accepted the result and that a payment was done. If a malicious buyer does not reveal the signature, the adjudicator, upon being requested by the seller, can verify that the seller fulfilled his delivery obligations and, in that case, extract a valid signature from the VES $\omega$. Similarly, if a malicious seller does not fulfill his delivery obligations, the adjudicator, upon being requested by the buyer, can tell the seller to fulfill them and, in the end, send the seller a valid signature. We refer to [29] for a detailed description. One of appealing properties of this transformation is that it adds very few overhead in terms of communication and computation.

Our PBSW protocol can also be extended to achieve fairness by applying this transformation to the POT scheme used as building block. In our protocol, the role of the adjudicator 
can be played by the judge. Both judge and buyers have to compute a key pair as defined in the VES scheme used and register the public key at the registration authority.

\section{EFFICIENCY}

The efficiency of our construction depends on the efficiency of the building blocks used to instantiate it. Efficiency measurements for the asymmetric watermark embedding technique we employ (algorithms SetupB, SetupV, Detect, Check) can be found in [9], which describes and implements an instantiation based on the homomorphic public key encryption scheme due to Paillier [23]. In [9], images of size 512 x 512 pixels are employed as digital content offered by $\mathcal{S}$, whose size after embedding the watermark in the encrypted domain is $536,870,912$ bits when each DCT coefficient is encrypted, or $6,318,080$ bits when composite signal representation is used. In the following, we employ watermarked messages $\left(m_{1}, \ldots\right.$, $\left.m_{N}\right)$ of those sizes as input to the POT scheme.

To evaluate the performance of the whole PBSW protocol, we implement the POT scheme proposed in [29] in a workstation equipped with an IntelCore2Duo processor at $3 \mathrm{GHz}$ and 4 Gbyte of RAM. All the functionalities are implemented in the $\mathrm{C}$ programming language. We use the PBC library ${ }^{2}$ for elliptic curve and pairing operations. We select type A pairings constructed on the curve $y^{2}=x^{3}+x$ over the field $F_{q}$ for a 512-bit prime $q=3 \bmod 4$. For other cryptographic primitives we employ the OpenSSL library ${ }^{3}$. Specifically, we employ RIPEMD-160 [32] as hash function and AES [33] in counter mode as block cipher.

The efficiency of the POT scheme in [29] in terms of computation and communication depends on the selection of three parameters: the number of messages $N$ offered by $\mathcal{S}$, the size of the watermarked messages, and the values $u$ and $l$ that define the maximum deposit allowed $D_{\max }=u^{l}$.

The performance of the initialization phase (algorithms POTInitB and POTInitS) depends on the number $N$ of messages and on the message size. Table I shows performance measurements when $N$ is 100,1000 and 10000 , and when the message size is $536,870,912$ and $6,318,080$ bits. As can be seen, the computation and communication complexity of both algorithms grows linearly with $N$, because POTInitS computes $N$ signatures and encrypts $N$ messages, and POTInitB verifies $N$ signatures. The overhead of the initial deposit sent from $\mathcal{B}$ to $\mathcal{S}$ is negligible.

The efficiency of the purchase phase does not depend on $N$, while the message size only affects algorithm POTComplete. However, it depends on the maximum deposit allowed $D_{\max }=u^{l}$, because this parameter determines the efficiency of a range proof that is computed by $\mathcal{B}$ to show that her account is non-negative. Table II shows the measurements of the purchase phase for a message size of $6,318,080$ bits and $N=100 . D_{\max }$ is 100,1000 and 10000 respectively, with fixed value $u=10$, and $1=2,3,4$ respectively. The value

\footnotetext{
${ }^{2}$ http://crypto.stanford.edu/pbc/

${ }^{3}$ http://www.openssl.org/
}

$l$ influences the computation and communication cost during POTReq () and POTVerReq ()${ }^{4}$

As can be seen, our protocol consists of an expensive initialization phase, whose cost grows linearly with the amount of messages and the message size, and very cheap purchase phases. In contrast, anonymous buyer-seller watermarking protocols consist of a cheap initialization phase and expensive transfer phases. Therefore, our protocol is more convenient in resource constrained settings because the initialization phase needs to be run only once, and later on a lot of very cheap purchase phases can be carried out.

An alternative to our PBSW protocol, which also preserves buyer's privacy by hiding from vendors which items are bought, would employ private information retrieval (PIR) [34]. Basically, the idea would be as follows. In the initialization phase, when the seller $\mathcal{S}$ computes $\left(c t_{1}, \ldots, c t_{N}\right)$, for $j=1$ to $N, \mathcal{S}$ picks randomly a key $K_{j}$ for a secure symmetric key encryption scheme, and encrypts $R_{j}=\operatorname{Enc}\left(K_{j}, c t_{j}\right)$. Keys $\left(K_{1}, \ldots, K_{N}\right)$ are given as input messages to the POT protocol, and $\left(R_{1}, \ldots, R_{N}\right)$ are given as input messages of the PIR scheme. To purchase a message, a buyer $\mathcal{B}$ obtains the symmetric key $K$ via the POT scheme, then obtains $R$ via the PIR scheme, and finally decrypts $R$ with $K$ to obtains $c t$. After that, this alternative would follow the PBSW protocol.

Since the size of the keys $K$ is smaller than the encrypted watermarked messages $c t$, the communication complexity of the initialization phase of the POT scheme decreases. However, after obtaining $K, \mathcal{B}$ has to perform a PIR query to obtain $R$. Most efficient computational PIR schemes achieve logsquared communication complexity in the database size [35], [36]. Therefore, if the number of purchases is expected to be small, this alternative would be more appealing. However, when the number of purchases is big, our PBSW protocol is more efficient since the communication complexity of purchase phases is constant. The exact threshold for the number of purchases in which our PBSW is more efficient depends on the concrete POT and PIR schemes used to instantiate both constructions.

\section{CONCLUSION}

We have proposed a privacy-preserving buyer-seller watermarking protocol, i.e., a protocol that allows copyright protection and in which buyers purchase from sellers without the seller learning the items they buy. Furthermore, we have also described how to extend the protocol to provide both buyers and sellers with optimistic fair exchange.

Further research needs to be conducted on the integration of privacy-preserving buyer-seller watermarking protocols with ecommerce and digital right management applications, as well as on the compliance of such applications with existing legislation. Another interesting goal is the design of lightweight privacy-preserving buyer-seller watermarking protocols suitable for resource-constrained devices.

\footnotetext{
${ }^{4}$ The value $u$ affects the efficiency of the initialization phase, but its influence is negligible when the message size is big. The most efficient choice in our case would be to fix $l=1$.
} 
TABLE I

IMPACT OF NUMBER OF MESSAGES AND MESSAGE SIZE ON THE PERFORMANCE OF THE POT INITIALIZATION PHASE

\begin{tabular}{cccccccc}
\multirow{2}{*}{ Algorithm } & $\begin{array}{c}\text { Message size } \\
\text { (bytes) }\end{array}$ & $\begin{array}{c}\text { Time } \\
\text { (seconds) }\end{array}$ & $\begin{array}{c}\text { Communication } \\
\text { (Kbytes) }\end{array}$ & $\begin{array}{c}\text { Time } \\
\text { (seconds) }\end{array}$ & $\begin{array}{c}\text { Communication } \\
\text { (Kbytes) }\end{array}$ & $\begin{array}{c}\text { Time } \\
\text { (seconds) }\end{array}$ & $\begin{array}{c}\text { Communication } \\
\text { (Kbytes) }\end{array}$ \\
\hline POTInitS() & \multirow{2}{*}{789,760} & 1.52 & 77,139 & 14.73 & 771,377 & 147.92 & $7,713,752$ \\
POTInitB() & & 0.37 & 0.015 & 3.31 & 0.015 & 32.94 & 0.015 \\
\hline POTInitS() & \multirow{2}{*}{$67,108,864$} & 91.89 & $6,553,614$ & 923.73 & $65,536,127$ & $9,209.55$ & $655,361,252$ \\
POTInitB() & 0.37 & 0.015 & 3.31 & 0.015 & 32.94 & 0.015
\end{tabular}

TABLE II

IMPACT OF MAXIMUM DEPOSIT ON THE PERFORMANCE OF THE POT PURCHASE PHASE

\begin{tabular}{lcccccc} 
& \multicolumn{2}{c}{$D_{\max }=\mathbf{1 0 0}$} & \multicolumn{2}{c}{$D_{\max }=\mathbf{1 0 0 0}$} & \multicolumn{2}{c}{$D_{\max }=\mathbf{1 0 0 0 0}$} \\
\cline { 2 - 7 } Algorithm & $\begin{array}{c}\text { Time } \\
(\mathrm{ms})\end{array}$ & $\begin{array}{c}\text { Communication } \\
\text { (bytes) }\end{array}$ & $\begin{array}{c}\text { Time } \\
(\mathrm{ms})\end{array}$ & $\begin{array}{c}\text { Communication } \\
\text { (bytes) }\end{array}$ & $\begin{array}{c}\text { Time } \\
(\mathrm{ms})\end{array}$ & $\begin{array}{c}\text { Communication } \\
\text { (bytes) }\end{array}$ \\
\hline POTReq() & 40 & 1,776 & 48 & 2,072 & 56 & 2,368 \\
POTVerReq() & 43 & - & 50 & - & 58 & - \\
POTResp() & 7.5 & 532 & 7.5 & 532 & 7.5 & 532 \\
POTVerResp () & 3.2 & - & 3.2 & - & 3.2 & - \\
POTComplete() & 11.7 & - & 11.7 & - & 11.7 & - \\
Total Purchase & 105.4 & 2,308 & 120.4 & $2, \overline{6}$ & 136.4 & 3,170
\end{tabular}

\section{REFERENCES}

[1] J. Tsai, S. Egelman, L. Cranor, and R. Acquisti, "The effect of online privacy information on purchasing behavior: An experimental study, working paper," June 2007.

[2] "Enforcing privacy promises: Section 5 of the ftc act," Federal Trade Commission Act, http://www.ftc.gov/privacy/privacyinitiatives/ promises.html.

[3] D. Boneh and J. Shaw, "Collusion-secure fingerprinting for digital data (extended abstract)," in CRYPTO, ser. Lecture Notes in Computer Science, D. Coppersmith, Ed., vol. 963. Springer, 1995, pp. 452-465.

[4] B. Pittzmann and M. Schunter, "Asymmetric fingerprinting," in $A d v$. in Cryptology - EUROCRYPT'96, ser. LNCS 1070, 1996, pp. 84-95.

[5] B. Pfitzmann and M. Waidner, "Anonymous fingerprinting," in EUROCRYPT, 1997, pp. 88-102.

[6] N. D. Memon and P. W. Wong, "A buyer-seller watermarking protocol," IEEE Transactions on Image Processing, vol. 10, no. 4, pp. 643-649, 2001.

[7] H.-S. Ju, H.-J. Kim, D.-H. Lee, and J.-I. Lim, "An anonymous buyerseller watermarking protocol with anonymity control," Information Security and Cryptology, pp. 421-432, Nov. 2002.

[8] C.-L. Lei, P.-L. Yu, P.-L. Tsai, and M.-H. Chan, "An efficient and anonymous buyer-seller watermarking protocol," IEEE Trans. on Image Processing, vol. 13, no. 12, pp. 1618-1626, 2004.

[9] M. Deng, T. Bianchi, A. Piva, and B. Preneel, "An efficient buyer-seller watermarking protocol based on composite signal representation," in Proceedings of the 11th ACM workshop on Multimedia and security. Princeton, New Jersey, USA: ACM New York, NY, USA, 2009, pp. 9-18.

[10] A. Rial, M. Kohlweiss, and B. Preneel, "Universally composable adaptive priced oblivious transfer," in Pairing, ser. Lecture Notes in Computer Science, H. Shacham and B. Waters, Eds., vol. 5671. Springer, 2009, pp. 231-247.

[11] D. Chaum, "Blind signatures for untraceable payments," in CRYPTO, 1982, pp. 199-203.

[12] R. Dingledine, N. Mathewson, and P. F. Syverson, "Tor: The secondgeneration onion router," in USENIX Security Symposium. USENIX, 2004, pp. 303-320.

[13] O. Berthold, H. Federrath, and M. Köhntopp, "Project "anonymity and unobservability in the internet"," in CFP '00: Proceedings of the tenth conference on Computers, freedom and privacy. New York, NY, USA: ACM, 2000, pp. 57-65.

[14] M. Gruteser and B. Hoh, "On the anonymity of periodic location samples," in $S P C$, ser. Lecture Notes in Computer Science, D. Hutter and M. Ullmann, Eds., vol. 3450. Springer, 2005, pp. 179-192.

[15] S. Kremer, Formal Analysis of Optimistic Fair Exchange Protocols. Université Libre de Bruxelles - Faculté des Sciences: Ph.D. Thesis, 2004.

[16] R. Canetti, "Universally composable security: A new paradigm for cryptographic protocols," in FOCS, 2001, pp. 136-145.
[17] W. Aiello, Y. Ishai, and O. Reingold, "Priced oblivious transfer: How to sell digital goods," in EUROCRYPT, ser. Lecture Notes in Computer Science, B. Pfitzmann, Ed., vol. 2045. Springer, 2001, pp. 119-135.

[18] A. Rial, M. Deng, T. Bianchi, A. Piva, and B. Preneel, "A provably secure anonymous buyer-seller watermarking protocol," IEEE Trans. on Information Forensics and Security.

[19] M. Barni and F. Bartolini, Watermarking Systems Engineering: Enabling Digital Assets Security and Other Applications, 1st ed. CRC Press, Boca Raton, February 2004.

[20] G. Tardos, "Optimal probabilistic fingerprint codes," J. ACM, vol. 55, no. 2, 2008.

[21] S. Goldwasser, S. Micali, and R. L. Rivest, "A digital signature scheme secure against adaptive chosen-message attacks," SIAM J. Comput., vol. 17, no. 2, pp. 281-308, 1988.

[22] S. Goldwasser and S. Micali, "Probabilistic encryption," J. Comput. Syst. Sci., vol. 28, no. 2, pp. 270-299, 1984.

[23] P. Paillier, "Public-key cryptosystems based on composite degree residuosity classes," in EUROCRYPT, 1999, pp. 223-238.

[24] I. Damgård and M. Jurik, "A generalisation, a simplification and some applications of paillier's probabilistic public-key system," in Public Key Cryptography, ser. Lecture Notes in Computer Science, K. Kim, Ed., vol. 1992. Springer, 2001, pp. 119-136.

[25] M. Bellare and O. Goldreich, "On defining proofs of knowledge," in CRYPTO '92, E. F. Brickell, Ed., vol. 740. Springer-Verlag, 1992, pp. $390-420$.

[26] J. Camenisch and M. Stadler, "Proof systems for general statements about discrete logarithms," Institute for Theoretical Computer Science, ETH Zürich, Tech. Rep. TR 260, Mar. 1997.

[27] J. Camenisch and V. Shoup, "Practical verifiable encryption and decryption of discrete logarithms," in CRYPTO, ser. Lecture Notes in Computer Science, D. Boneh, Ed., vol. 2729. Springer, 2003, pp. 126-144.

[28] G. Poupard and J. Stern, "Fair encryption of rsa keys," in EUROCRYPT, 2000, pp. 172-189.

[29] A. Rial and B. Preneel, "Optimistic fair priced oblivious transfer," in AFRICACRYPT, ser. Lecture Notes in Computer Science, D. J. Bernstein and T. Lange, Eds., vol. 6055. Springer, 2010, pp. 131-147.

[30] N. Asokan, V. Shoup, and M. Waidner, "Optimistic fair exchange of digital signatures (extended abstract)," in EUROCRYPT, 1998, pp. 591606.

[31] D. Boneh, C. Gentry, B. Lynn, and H. Shacham, "Aggregate and verifiably encrypted signatures from bilinear maps," in EUROCRYPT, ser. Lecture Notes in Computer Science, E. Biham, Ed., vol. 2656. Springer, 2003, pp. 416-432.

[32] H. Dobbertin, A. Bosselaers, and B. Preneel, "RIPEMD-160: A strengthened version of RIPEMD," in FSE, 1996.

[33] NIST, Advanced Encryption Standard (AES) (FIPS PUB 197), National Institute of Standards and Technology, 2001.

[34] E. Kushilevitz and R. Ostrovsky, "Replication is not needed: Single database, computationally-private information retrieval," in FOCS, 1997, pp. 364-373. 
[35] H. Lipmaa, "An oblivious transfer protocol with log-squared communication," in ISC, ser. Lecture Notes in Computer Science, J. Zhou, J. Lopez, R. H. Deng, and F. Bao, Eds., vol. 3650. Springer, 2005, pp. 314-328.

[36] C. Gentry and Z. Ramzan, "Single-database private information retrieval with constant communication rate," in ICALP, ser. Lecture Notes in Computer Science, L. Caires, G. F. Italiano, L. Monteiro, C. Palamidessi, and M. Yung, Eds., vol. 3580. Springer, 2005, pp. 803-815.

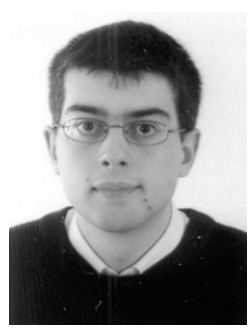

Alfredo Rial received his master's degree in Telecommunication Engineering from the Universidade de Vigo, Spain, in 2008. Currently he is a $\mathrm{Ph} . \mathrm{D}$. candidate at Katholieke Universiteit Leuven, Belgium, under the supervision of Prof. Bart Preneel. $\mathrm{He}$ is funded by the Research Foundation - Flanders (FWO). His research interests include public key cryptography, cryptographic protocols design and privacy.

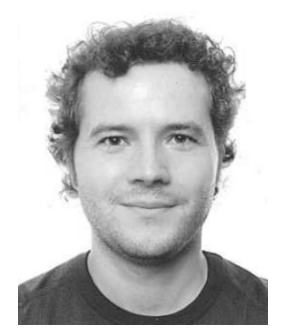

Josep Balasch received his master's degree in Telecommunication Engineering from the Universitat Politecnica de Catalunya, Barcelona, Spain, in 2008. Currently he is pursuing a Ph.D. degree at the Katholieke Universiteit Leuven, Belgium. His research interests include efficient implementations of cryptographic algorithms, embedded security and privacy-preserving systems.

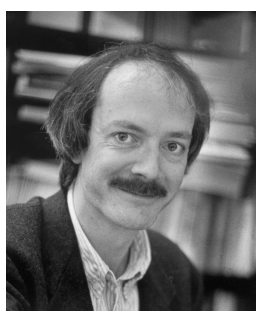

Bart Preneel (Member, IEEE) received the M.S degree in electrical engineering and the Ph.D. degree in applied sciences (cryptology) from the Katholieke Universiteit Leuven, Belgium, in 1987 and 1993, respectively. $\mathrm{He}$ is currently Full Professor with the Katholieke Universiteit Leuven. He was Visiting Professor at five universities in Europe and was a Research Fellow with the University of California at Berkeley. He has authored and coauthored more than 300 reviewed scientific publications and is the inventor of three patents. His main research interests are cryptography and information security. Prof. Preneel is President of the International Association for Cryptologic Research (IACR) and of the Leuven Security Excellence Consortium (L-SEC vzw.), an association of 60 companies and research institutions in the area of e-security. 